\title{
Low rank or nuclear-norm minimization: Are we solving the right problem?
}

\author{
Yuchao Dai, Hongdong Li \\ College of Engineering and Computer Science \\ The Australian National University, Canberra, ACT \\ Email: \{yuchao.dai, hongdong.li\}@ anu.edu.au
}

\begin{abstract}
Low rank method or rank-minimization has received considerable attention from recent computer vision community. Due to the inherent computational complexity of rank problems, the non-convex rank function is often relaxed to its convex relaxation, i.e. the nuclear norm. Thanks to recent progress made in the filed of compressive sensing (CS), vision researchers who are practicing CS are fully aware, and conscious, of the convex relaxation gap, as well as under which condition (e.g. RIP) the relaxation is tight (i.e. with nil gap).

In this paper, we however wish to alert the potential users of the low-rank method that: focusing too much on the issue of relaxation gap and optimization may possibly adversely obscure the "big picture" of the original computer vision problem. In particular, this paper shows that for many commonly cited lowrank problems, the original rank-minimization objective function do not necessarily lead to the desired solution. Degenerate solutions and multiplicity seem often or always exist. Even if a certain nuclear-norm solution is a provably tight relaxation, this solution can possibly be meaningless in its particular context. We therefore advocate that, in solving vision problems via low-rank method, special care must be given, and domain-dependent prior knowledge must be taken into account. This paper summarizes recent relevant theoretical results, provides original analysis, uses real examples to demonstrate the practical implications. As an example, we also provide our explanation to recent success of using nuclear norm without paying duly attention to the above alert.
\end{abstract}

\section{BACKGROUND}

Recently, low rank problems, e.g. affine constrained rank minimization problem (ARMP), have received considerable attention from the computer vision communities, among many other research communities in science and in engineering. In the area of computer vision, a wide range of problems can be, and have been, represented under this low-rank or rank-minimization framework. Low rank or sparse representation models have been used to interpret/approximate in high dimension and for large scale measurements. The low rank formulation seems to be able to effectively capture the loworder structure of the underlying problems.

It is well-known that rank problems are generally extremely hard to solve (, and also hard to approximate. c.f. [1]). Thanks to recent progress in compressive sensing, now we are equipped with efficient tools to solve (approximately) many of the rank-related minimization problems using effective convex relaxation, which otherwise are generally NP-hard to solve. Successful examples of applying this low-rank scheme abound in computer vision, see for example, subspace segmentation [2], low rank texture repairing [3], video restoration [4], rigid structure from motion [5] and non-rigid structure from motion [6], robust principle component analysis [7] etc. Table I gives an incomplete list of recent works of using rank minimization in computer vision.

A common trick, that is widely used to solve the otherwise NP-hard rank problem, is to replace (relax) the rank objective function by nuclear-norm. Such a relaxation is shown to be very successful in solving many rank problems. Recent results from compressive sensing (CS) also confirm that, if certain conditions (such as RIP etc. ) are satisfied, the nuclear-norma minimization solution will lead to the exact solution to the original rank-minimization problem. In other words, under that certain conditions, the relaxation gap is zero, and these two problems (rank versus nuclear norm) are equivalent. This is an important theoretical result, and encouraging. Investigation into what these conditions are, as well as how to guarantee these conditions in certain practical application context have been one of the research focuses.

However, in this paper, we focus on a very different aspect of the low-rank/nuclear-norm paradigm. Based on carefully analyzing various low-rank problems in vision, we have gained much insights into the structures of low-rank problems. And we wish to use this paper to alert potential users of the lowrank methods that: when facing a particular low-rank problem in computer vision, you need to think carefully about: Rank, or nuclear-norm, are we solving the right problem ?

\section{A. Some Examples}

Despite the overall success of the use of low-rank and nuclear norm technique, some recent works indeed show that, sometimes the rank-minimization formulation is not sufficient in solving certain problems, and does not necessarily lead to the desired solution.

Example 1: In the low-rank texture repairing work [3], Liang et al. concluded that "although being low-rank is a necessary condition for most regular, structured images, it is certainly not sufficient". To give an example, Fig. 1 shows three texture images-all of which have exactly the same and low rank ${ }^{1}$, though the three have significantly different visual appearances. Solving a plain low-rank problem exactly does not necessarily lead to the desired low rank repair, extra domain-dependent prior or assumption must be enforced.

\footnotetext{
${ }^{1}$ This is because column-wise or row-wise re-order of a matrix does not change its rank.
} 
TABLE I. AN INCOMPLETE LIST OF RANK MINIMIZATION PROBLEMS IN COMPUTER VISION.

\begin{tabular}{|c|c|c|}
\hline Problem & Rank Minimization Formulation & Convex Relaxation \\
\hline Projective Factorization [5], [8] & $\min _{\mathrm{W}, \Lambda} \operatorname{rank}(\mathrm{W}), \mathrm{W}=\Lambda \odot \mathrm{M}$ & $\min \|\mathrm{W}\|_{*}$ \\
\hline Non-rigid Structure from Motion [6], [9] & $\min _{\mathrm{S}} \operatorname{rank}\left(\mathrm{S}^{\sharp}\right), \mathrm{W}=\mathrm{RS}, \mathrm{S}^{\sharp}=\mathrm{S}$ & $\min \left\|S^{\sharp}\right\|_{*}$ \\
\hline TILT [10] & $\min _{\mathrm{I}^{0}, \mathrm{E}, \tau} \operatorname{rank}\left(\mathrm{I}^{0}\right)+\gamma\|\mathrm{E}\|_{0}, \mathrm{I} \circ \tau=\mathrm{I}^{0}+\mathrm{E}$ & $\min _{\mathrm{I}^{0}, \mathrm{E}, \tau}\left\|\mathrm{I}^{0}\right\|_{*}+\gamma\|\mathrm{E}\|_{1}$ \\
\hline RASL [11] & $\min _{\mathrm{A}, \mathrm{E}, \tau} \operatorname{rank}(\mathrm{A})+\gamma\|\mathrm{E}\|_{0}, \mathrm{D} \circ \tau=\mathrm{A}+\mathrm{E}$ & $\min _{\mathrm{A}, \mathrm{E}, \tau}\|\mathrm{A}\|_{*}+\lambda\|\mathrm{E}\|_{1}$ \\
\hline Calibration [12] & $\min \operatorname{rank}\left(\mathrm{A}_{i}\right)+\lambda\left\|\mathrm{E}_{i}\right\|_{0}, \mathrm{I}_{0} \circ\left(\tau_{0}, \tau_{i}\right)^{-1}=\mathrm{A}_{i}+\mathrm{E}_{i}$ & $\min \left\|\mathrm{A}_{i}\right\|_{*}+\lambda\left\|\mathrm{E}_{i}\right\|_{1}$ \\
\hline Radiometric calibration [13] & $\hat{g}=\arg \min _{g} \operatorname{rank}(\mathrm{A}), \mathrm{A}=g \circ \mathrm{D}$ & condition number \\
\hline Subspace Segmentation [2] & $\min \operatorname{rank}(\mathrm{Z})+\lambda\|\mathrm{E}\|_{0}, \mathrm{X}=\mathrm{AZ}+\mathrm{E}$ & $\min \|\mathrm{Z}\|_{*}+\lambda\|\mathrm{E}\|_{1}$ \\
\hline Photometric Stereo [14] & $\min \operatorname{rank}(\mathrm{A})+\gamma\|\mathrm{E}\|_{0}, \mathrm{D}=\mathrm{A}+\mathrm{E}$ & $\min \|\mathrm{A}\|_{*}+\lambda\|\mathrm{E}\|_{1}$ \\
\hline Euclidean Structure from Motion [15] & $\min _{Z_{k i}^{*} \leq 1} \operatorname{rank}\left[\mathrm{H}_{y 1 N}, \cdots, \mathrm{H}_{y N-1 N}\right]$ & LMIRank [16] \\
\hline Low-rank Texture Repairing [3] & $\min _{\mathrm{I}} \operatorname{rank}(\mathrm{I}), I_{i j}=D_{i j}$ & $\min _{\mathrm{I}}\|\mathrm{I}\|_{*}+\lambda\|\mathrm{W}\|_{1}$ \\
\hline Correspondence Matching [17] & 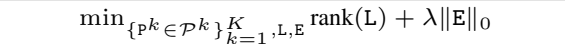 & $\min _{\left\{\mathrm{P} k \in \mathcal{P}^{k}\right\}_{k=1}^{K, \mathrm{~L}, \mathrm{E}}}\|\mathrm{L}\|_{*}+\lambda\|\mathrm{E}\|_{1}$ \\
\hline
\end{tabular}
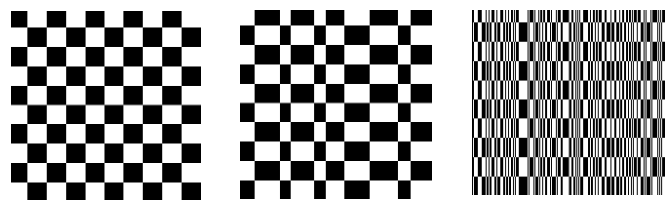

Fig. 1. An example of rank's insufficiency. These three images have exactly the same rank, but they go from purely regular, to nearly regular, and to nearly random textures [3].

Example 2: Angst-Rach-Pollefeyes [5] and Dai-Li-He [8] both formulated the projective structure-from-motion problem (Under the perspective imaging model $\Lambda\left[\mathrm{x}_{i j}\right]=\mathrm{PX}$, recover $\mathrm{P}, \mathrm{X}$ and $\Lambda$ with the sole input of $\mathrm{x}_{i j}$ ) as rank minimization and solve it via (generalized) nuclear-norm minimization. Although the nuclear-norm minimization always give rises to a unique solution, one must be fully conscious of that: this solution needs not be the correct, or the desirable solution in the context of SfM.

Spurious solutions always exist, and may happen to be found by solving the rank-minimization formulation. For instance, the well-known degenerate case or critical configurations (see [18] ) are low-rank scenarios, which satisfy the low-rank condition mathematically perfectly; they are however not the desired solution. In Fig. 2, we show the example of conjugate configuration from [18], where the two configuration corresponds to identical images however the reconstruction is not projectively equivalent.

Recently, Nasihatkon et al. [19] presented a generalized projective reconstruction theorem, which shows that projective reconstruction is possible under a much weaker restriction than requiring all estimated projective depths to be nonzero. Furthermore, they presented a class of depth constraints under which any reconstruction of cameras and points projecting into given image points is projectively equivalent to the true camera-point configuration.

Example 3: In non-rigid structure-from-motion (NRSfM), a recent work by Dai et al. [9] showed that under rank minimization formulation to NRSfM factorization

$$
\min \operatorname{rank}\left(\mathrm{S}^{\sharp}\right) \text {, s.t. } \mathrm{W}=\mathrm{RS}, \mathrm{S}^{\sharp}=g(\mathrm{~S})
$$

there exist configurations such that multiple solutions explain the same measurements exactly (Theorem 6).

Example 4: Latent low rank representation (LatLRR)
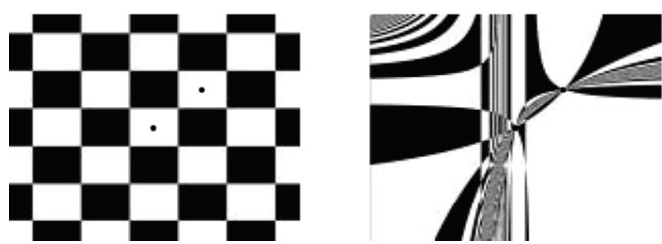

Fig. 2. Two conjugate configurations of points on the critical quadric $Z=$ $X Y$ viewed in the direction of the $z$-axis. Camera centers are drawn with black circles. [18].

model:

$$
\min _{\mathrm{Z}, \mathrm{L}} \operatorname{rank}(\mathrm{Z})+\operatorname{rank}(\mathrm{L}), \text { s.t. } \mathrm{X}=\mathrm{XZ}+\mathrm{LX} .
$$

The nuclear norm minimization formulation for LatLRR:

$$
\min _{\mathrm{Z}, \mathrm{L}}\|\mathrm{Z}\|_{*}+\|\mathrm{L}\|_{*} \text {, s.t. } \mathrm{X}=\mathrm{XZ}+\mathrm{LX} .
$$

By analyzing the noiseless LatLRR model, Zhang et al. [20] showed that even for this simple rank minimization problem the validity of replacing rank minimization with nuclear norm minimization may still break down. Furthermore, they proved that the solution to the nuclear norm minimization formulation of LatLRR is non-unique.

Example 5: In the low-rank subspace segmentation work, Lu et al. [21] gives the following simple example to show the insufficiency of rank in subspace segmentation.

Let $\mathrm{X}_{1}=\left[\begin{array}{ll}1 & 2 \\ 0 & 0\end{array}\right]$ and $\mathrm{X}_{2}=\left[\begin{array}{ll}0 & 0 \\ 1 & 2\end{array}\right]$ be some data points drawn from subspaces $\mathcal{S}_{1}$ and $\mathcal{S}_{2}$, respectively. We assume $\operatorname{dim}\left(\mathcal{S}_{1}\right)=\operatorname{dim}\left(\mathcal{S}_{2}\right)=1$, thus $\mathcal{S}_{1}$ and $\mathcal{S}_{2}$ are orthogonal, and $\operatorname{rank}\left(\mathrm{X}_{1}\right)=\operatorname{rank}\left(\mathrm{X}_{2}\right)=1$. Let $\mathrm{X}=\left[\mathrm{X}_{1}, \mathrm{X}_{2}\right]$, the solutions to the low rank representation problem $(\mathrm{X}=\mathrm{XZ})$ are not unique and one of which is:

$$
\mathrm{z}^{*}=\left[\begin{array}{cccc}
0.5 & 1 & 1 & 2 \\
0.25 & 0.5 & -0.5 & -1 \\
1 & 2 & 0.5 & 1 \\
-0.5 & -1 & -0.25 & 0.5
\end{array}\right]
$$

This example shows that solution to the original low rank representation model does not guarantee to be block diagonal, thus can not be used to recover the true segmentation. In a related work, Shi and Yu [22] showed that nuclear norm minimization may lead to undesirable matrix despite being low rank. 
Motivated by the above examples, in this work we revisit the rank-nuclear-norm formulations used in various vision problems. Our main intention is to provide careful analysis to the low-rank problem formulation, from a novel perspective. In particular, we focus on the uniqueness (or multiplicity) property of the original low-rank or rank-minimization formulation, as well as that of the convex relaxed nuclear-norm formulation. We will show that, while low-rank is often a necessary condition for the correct solution of many vision problems, using rank-minimization objective alone is not sufficient to yield the desired solution. Multiple solutions can happen, and in fact, will almost always happen for many of our commonly used low-rank formulation in recent vision application. If this situation happens, then, even when the relaxation gap caused by the nuclear-norm relaxation approaches zero (i.e. , exact recovery), we still cannot be sure whether or not the found solution is the correct solution that we are after. Moreover, this paper also attempts to provide a possible explanation to the apparent success of nuclear-norm in various applications. Specifically, we show that, the relaxed nuclear norm formulation may possess some other (nice) property that is not in the original low-rank formulation, and it may be this property that better "rectifies" or "regularizes" the problem, and achieve good and unique solution.

It is our hope that, by presenting these in-depth (and novel) theoretical analysis and practical discussions, this paper will provide useful service to the community.

a) Organization.: This paper is organized as follows. In Section-2, we provide basic mathematical definition/formulations to the low-rank, and nuclear-norm problems . In Section-3, we present a recent and very relevant result in compressive sensing, regarding the uniqueness analysis for the low-rank problems under different constraints. This result serves the central theoretical foundation of this paper. In Section-4, we will use "low-rank representation" (or "dictionary learning") as a typical example of low-rank problems, to illustrate the complex structure of the (multiplicity) solution space. Section-5 demonstrate possible ways to incorporate prior knowledge to better regularize the problem, thus better confines the solution space. Section-6 is devoted to practical implications of the results of this paper. We will, again, use a set of concrete examples of low-rank problem, from computer vision applications, to convey our main points. Then is the conclusion Section.

\section{PROBLEMS FORMULATIONS}

In this section, we give the formulations of typical low-rank or rank-minimization problems, as well as the nuclear-norm relaxation.

\section{A. Affine constrained rank minimization}

The affine constrained rank minimization problem (ARMP) aims at finding a matrix of minimum rank that satisfies a given system of linear equality constraints, i.e. ,

$$
\text { (P1): } \min \operatorname{rank}(\mathrm{x}) \text {, s.t. }, \mathcal{A}(\mathrm{x})=\mathrm{b},
$$

where $\mathcal{A}: \mathbb{R}^{n_{1} \times n_{2}} \rightarrow \mathbb{R}^{m}$ is a linear operator. The above linear mapping can always be expressed in its matrix representation form as $\mathcal{A}(\mathrm{X})=\operatorname{Avec}(\mathrm{X})$, where $\operatorname{vec}()$ denotes the vector of $\mathrm{X}$ with its columns stacked in order on top of one another, and $\mathrm{A}$ is an $m \times n_{1} n_{2}$ matrix.

There is also another group of problems pursuit not only a rank minimization solution but also require the solution to be positive semi-definite (PSD), leading to PSD-constrained rank-minimization [23]:

$$
\text { (P2) : } \quad \min \operatorname{rank}(\mathrm{X}) \text {, s.t. } \operatorname{Avec}(\mathrm{X})=\mathbf{b}, \mathrm{X} \succeq 0 .
$$

\section{B. Nuclear-norm relaxation}

The rank-minimization problem $(\mathbf{P} 1$ or $\mathbf{P 2})$ is generally NP-hard [1]. A common trick to solve it approximately is to replace the rank function with the nuclear norm of the matrix, defined as $\|\mathrm{X}\|_{*}=\sum_{i=1}^{r} \sigma_{i}(\mathrm{X})$ where $\sigma_{i}(\mathrm{X})$ are the singular values and $r=\operatorname{rank}(\mathrm{X})^{2}$.

With such relaxation in use, the above rank problems are approximated by the following nuclear-norm problems:

$$
\begin{gathered}
(\mathbf{P 3}): \min \|\mathrm{X}\|_{*}, \text { s.t. }, \operatorname{Avec}(\mathrm{X})=\mathbf{b} . \\
(\mathbf{P 4}): \quad \min \operatorname{trace}(\mathrm{X}) \text {, s.t. }, \operatorname{Avec}(\mathrm{X})=\mathbf{b}, \mathrm{X} \succeq 0 .
\end{gathered}
$$

\section{UNIQUENESS CONDITION ANALYSIS}

When we use the nuclear-norm relaxation to solve an original rank-minimization problem, a key theoretical question that naturally arises is that: can the nuclear-norm relaxation always recover the exact rank-minimization solution, or is the relaxation gap tight? Conventionally, this kind of "gap questions" are answered through some statistical analysis of the incoherent conditions, or Restricted isometry property (RIP), etc. . developed in the field of compressive sensing. Recht, Fazel and Parrilo [1] extended it to matrix rank minimization problem and proposed to use Restricted Isometry Property (RIP) to characterize the condition of unique recovery. However RIP is not invariant with respect to any invertible map, and relies crucially on particular stochastic assumption about the measurement matrix (e.g. with Gaussian entries).

In this section, we take a different, and more general, viewpoint to look at the problem. As for any relaxation method, while we need to characterize the properties of the nuclearnorm relaxation itself, we also need to analyze when the original problem of rank-minimization admit unique solution. In fact, in the ARMP setting, the ability to recover $\mathrm{X}$ from $\mathcal{A}(\mathrm{X})=\mathbf{b}$ depends exclusively on the null-space of $\mathcal{A}$, which is invariant under invertible map.

Based on the analysis of the null-space property of $\mathcal{A}$, Eldar, Needell and Plan [24] gives the following theorem regarding the uniqueness of rank minimization problem P1.

Theorem III.1. When $\mathrm{X}$ and $\mathrm{X}^{\prime}$ are each rank-r, then $\left(\mathrm{X}-\mathrm{X}^{\prime}\right)$ is at most rank-2r. To ensure that (1) reconstruct all rank-r matrices, a necessary and sufficient condition is that there are no rank-2r (or less) matrices in the null-space of $\mathcal{A}$.

Here we provide our simplified proof.

\footnotetext{
${ }^{2}$ Nuclear norm of $\mathrm{X}$, i.e., $\|\mathrm{X}\|_{*}$ is the convex envelope of $\operatorname{rank}(\mathrm{x})$ on the set $\left\{\mathrm{X} \in \mathbb{R}^{m \times n}:\|\mathrm{X}\| \leq 1\right\}$, where $\|\mathrm{X}\|$ is the operator norm, i.e. the largest singular value of $\mathrm{X}$.
} 
Proof: Sufficiency: Suppose there are no rank- $2 r$ or less matrices in the null space of $\mathcal{A}$, if the rank minimization formulation is not unique, i.e. , $\mathrm{x}_{1}$ and $\mathrm{x}_{2}$ achieve identical measurements $\mathcal{A}\left(\mathrm{X}_{1}\right)=\mathcal{A}\left(X_{2}\right)$ with rank $r$, then $\left(\mathrm{X}_{1}-\mathrm{X}_{2}\right)$ lies in the null space of $\mathcal{A}$, additionally $\operatorname{rank}\left(\mathrm{X}_{1}-\mathrm{X}_{2}\right) \leq 2 r$, which conflicts the assumption. Therefore, (1) reconstructs all rank- $r$ matrices if there are no rank- $2 r$ or less matrices in the null space of $\mathcal{A}$.

Necessity: Suppose (1) reconstructs all rank- $r$ matrices, i.e. the rank minimization solution is unique, if there is a rank-2r or less matrices $\mathrm{Y}$ in the null space of $\mathcal{A}$, then we can always construct two rank- $r$ matrices $\mathrm{X}_{1}$ and $\mathrm{x}_{2}$ such that $\mathcal{A}\left(\mathrm{x}_{1}\right)=\mathcal{A}\left(\mathrm{x}_{2}\right)$. One easy way is: Denote the singular value decomposition (SVD) of $\mathrm{Y}$ as $\mathrm{Y}=\mathrm{U}_{Y} \Sigma_{Y} \mathrm{~V}_{Y}^{T}, \quad \Sigma_{Y}=\operatorname{diag}\left(\sigma_{1}, \sigma_{2}, \cdots, \sigma_{2 r}\right)$, by letting $\Sigma_{X_{1}}=\operatorname{diag}\left(\sigma_{1}, \sigma_{2}, \cdots, \sigma_{r}\right), 0, \cdots, 0$ and $\Sigma_{X_{2}}=$ $\operatorname{diag}\left(0, \cdots, 0,-\sigma_{r+1},-\sigma_{r+2}, \cdots,-\sigma_{2 r}\right)$, we obtain $\mathrm{X}_{1}=$ $\mathrm{U}_{Y} \Sigma_{X_{1}} \mathrm{~V}_{Y}^{T}$ and $\mathrm{X}_{2}=\mathrm{U}_{Y} \Sigma_{X_{2}} \mathrm{~V}_{Y}^{T}$ such that $\operatorname{rank}\left(\mathrm{X}_{1}\right)=$ $\operatorname{rank}\left(\mathrm{X}_{2}\right)=r$ and $\mathcal{A}\left(\mathrm{X}_{1}\right)=\mathcal{A}\left(\mathrm{X}_{2}\right)$. We conclude: if (1) reconstruct all rank- $r$ matrices, then there are no rank- $2 r$ or less matrices $\mathrm{Y}$ in the null space of $\mathcal{A}$,

Figure III illustrates the relationship between rank- $r$ manifold and rank- $2 r$ manifold. Let two rank- $r$ matrices be $\mathrm{X}_{1}$ and $\mathrm{X}_{2}$, then $\mathrm{Y}_{1}=\mathrm{X}_{1}-\mathrm{X}_{2}$ lies in the matrix manifold $\operatorname{rank}(\mathrm{Y}) \leq 2 r$. Note that, given a matrix $\mathrm{Y}_{2}$ in the matrix manifold $\operatorname{rank}(\mathrm{Y}) \leq 2 r$, we can always construct pairs of rank$r$ matrices $\mathrm{X}_{3}$ and $\mathrm{X}_{4}$ or $\mathrm{X}_{5}$ and $\mathrm{X}_{6}$ such that $\mathrm{Y}_{2}=\mathrm{X}_{3}-\mathrm{X}_{4}$ and $\mathrm{Y}_{2}=\mathrm{X}_{5}-\mathrm{X}_{6}$. It is worth to note that the relationship is not necessarily full coverage, i.e., it is possible that some matrix $\mathrm{X}$ with $\operatorname{rank}(\mathrm{X})=r$ can not be reached from any matrix $\mathrm{Y}$ with $\operatorname{rank}(\mathrm{Y}) \leq 2 r, \mathrm{Y} \neq 0$ such that $\operatorname{rank}(\mathrm{X}+\mathrm{Y})=r$.

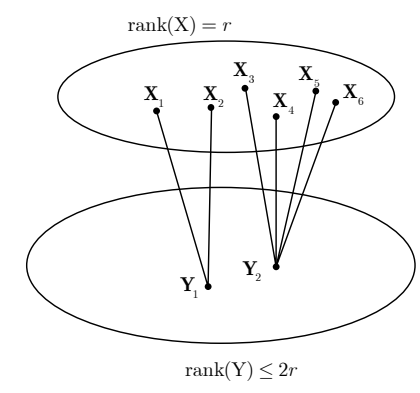

Fig. 3. Illustration of the relationship between rank- $r$ matrix manifold and rank- $2 r$ matrix manifold.

\section{A. Positive semi-definite low rank recovery}

Positive-semi-definiteness (PSD) constraint is also common in vision applications, e.g. , in various kernel methods, or where there is distance matrices. For PSD constrained rank minimization, Oymak and Hassibi [25] presented the PSD weak threshold and strong threshold.

Theorem III.2. Let $\mathrm{X} \in \mathbb{S}_{+}^{n}$ be a rank $r$ matrix with eigenvalue decomposition (EVD) as $\mathrm{X}=\mathrm{U} \Lambda \mathrm{U}^{T}$ and $\Lambda \in \mathbb{R}^{r \times r}$. Then $\mathrm{X}$ is the unique minimizer if for all $\mathrm{W} \in \mathcal{N}(\mathrm{A})$, we have: $\mathrm{W}$ is not hermittian or $\operatorname{trace}(\mathrm{W})>0$ or $\eta_{-}\left(\overline{\mathrm{U}}^{T} \mathrm{~W} \overline{\mathrm{U}}\right)>0$, where $[\mathrm{U}, \overline{\mathrm{U}}]$ is a unitary matrix.

We include this PSD result here mainly for the sake of completeness, though we did not explore this topic further in this paper.

\section{CHARACTERIZING THE LOW-RANK SOLUTION SPACE}

In this section, we use low-rank representation (i.e. Problem $\mathbf{P 5}$ to be given below) as an example to give detailed analysis to the structure of the solution space (when even multiple solution exist) of the rank minimization approach in computer vision.

\section{A. Low rank representation}

In subspace segmentation [2], multi-view computer vision [6], multi-task learning [26] and etc, there is a wide range of problems all share the following low-rank representation formulation:

$$
\text { (P5 :) } \quad \min \operatorname{rank}(\mathrm{Z}) \text {, s.t. }, \mathrm{W}=\mathrm{AZ},
$$

i.e. , finding the rank minimum solution to a matrix equation system, where $\mathrm{A} \in \mathbb{R}^{m \times n}, \mathrm{Z} \in \mathbb{R}^{n \times p}$ and $\mathrm{W} \in \mathbb{R}^{m \times p}, m<n$.

By using the nuclear norm relaxation, we obtain

$$
\text { (P6 :) } \min \|\mathrm{Z}\|_{*} \text {, s.t. }, \mathrm{W}=\mathrm{AZ} \text {. }
$$

Through introducing matrix vectorization operator and Kronecker product, the above matrix equation can be equivalently expressed as:

$$
\operatorname{vec}(\mathrm{W})=\left(\mathrm{I}_{p} \otimes \mathrm{A}\right) \operatorname{vec}(\mathrm{Z})=\mathrm{A}^{\star} \operatorname{vec}(\mathrm{Z}),
$$

where $\mathrm{I}_{p} \in \mathbb{R}^{p \times p}$ denotes the identity matrix. It is easy to check that the problem (5) falls into the family of affine rank minimization problem (1).

\section{B. A closed-form solution}

For the low rank representation problem (P5), Liu et al. [2] proposed the following theorem regarding its nuclear-norm relaxation:

Theorem IV.1. Suppose the matrix A has full row rank, then $\mathrm{Z}^{*}=\mathrm{A}^{\dagger} \mathrm{W}$ is the unique minimizer to the nuclear norm minimization problem (6), where $\mathrm{A}^{\dagger}$ is the pseudo-inverse of $\mathrm{A}$. Additionally, $\operatorname{rank}\left(\mathrm{Z}^{*}\right)=\operatorname{rank}\left(\mathrm{Z}_{0}\right)$ is also a globally optimal solution to rank minimization problem (5).

Theorem IV.1 shows the uniqueness of the nuclear norm minimization relaxation. However the original rank minimization problem may still have multiple solutions. Here we give analysis to P5. Through the analysis of null space, we prove that there exists multiple solutions for the rank minimization problem, additionally, we give the explicit representation of the rank minimization subspace. Finally, we show how to exploit the rank minimization subspace to achieve rank minimization solution with desired property.

\section{Null space analysis}

First, we introduce singular value decomposition of Kronecker product as:

Lemma IV.2. [27] If $\mathrm{C}=\mathrm{A} \otimes \mathrm{B}$, then the singular value decomposition (SVD) of $\mathrm{C}$ can be computed as:

$$
\mathrm{C}=\left(\mathrm{U}_{A} \Sigma_{A} \mathrm{~V}_{A}^{T}\right) \otimes\left(\mathrm{U}_{B} \Sigma_{B} \mathrm{~V}_{B}^{T}\right)=\left(\mathrm{U}_{A} \otimes \mathrm{U}_{B}\right)\left(\Sigma_{A} \otimes \Sigma_{B}\right)\left(\mathrm{V}_{A} \otimes \mathrm{V}_{B}\right)^{T},
$$


where the SVD of $\mathrm{A}$ and $\mathrm{B}$ are $\mathrm{A}=\mathrm{U}_{A} \Sigma_{A} \mathrm{~V}_{A}^{T}$ and $\mathrm{B}=\mathrm{U}_{B} \Sigma_{B} \mathrm{~V}_{B}^{T}$ respectively.

If the null space of $\mathrm{B}$ is $\mathcal{N}(\mathrm{B})$, the null space of $\mathrm{C}$ can be expressed as $\mathrm{V}_{A} \otimes \mathcal{N}(\mathrm{B})$.

From Lemma (IV.2), we can conclude that the null space of $A^{\star}$ can be expressed as $I_{p} \otimes \mathcal{N}(A)$. Generally, dimension of null space of A is $n \times(n-m)$, therefore the dimension of null space $\mathrm{A}^{\star}$, i.e. $\mathrm{I}_{p} \otimes \mathcal{N}(\mathrm{A})$ is $n p \times(n-m) p$. When transforming each column to the corresponding matrix form $\mathrm{Z}_{i}, i=1, \cdots, p(n-$ $m$ ) of dimension $n \times p, \mathrm{AZ}_{i}=0$. Meanwhile each $\mathrm{Z}_{i}$ has only one column with non-zeros elements, therefore $Z_{i}$ is of rank 1 .

Theorem IV.3. Assume $\mathrm{A} \neq 0$ and $\mathrm{W}=\mathrm{AZ}$ has feasible solutions, i.e. , $\mathrm{W} \in \operatorname{span}(\mathrm{A})$. Then the nuclear norm minimization can not recover all the rank-r matrices.

Proof: As we have shown above, we have found a group of orthogonal basis of the null space of $A^{\star}$, where each basis $\mathrm{Z}_{i}$ is rank-1 matrix. Therefore there exists rank-1 matrices solution in the null space of $A^{\star}$.

According to Theorem III.1, the nuclear norm minimization formulation (3) can not recover all matrices $Z_{0}$ with $\operatorname{rank}\left(\mathrm{Z}_{0}\right) \leq r$.

\section{Characterizing the solution subspace}

The pseudo-inverse solution to $\mathrm{W}=\mathrm{AZ}$ is $\mathrm{Z}^{*}=\mathrm{A}^{\dagger} \mathrm{W}$. Assume the rows of A are linearly independent (so that $m \leq n$ ), then $\mathrm{A}^{\dagger}=\mathrm{A}^{T}\left(\mathrm{AA}^{T}\right)^{-1}$. Therefore $\mathrm{Z}^{*}$ lies in the range of $\mathrm{A}^{T}$. The null space of $\mathrm{A}$ is $\mathcal{N}(\mathrm{A})$.

The solution space to the homogeneous equation $\mathrm{AZ}=0$ can be expressed as $\mathrm{Z}=\mathcal{N}(\mathrm{A}) \mathrm{C}$, where $\mathrm{C} \in \mathbb{R}^{n-m) \times p}$ is the coefficient matrix.

Given the pseudo-inverse solution $\mathrm{A}^{\dagger} \mathrm{W}$ and the null space $\mathcal{N}(\mathrm{A})$, all the solutions to $\mathrm{W}=\mathrm{AZ}$ can be expressed as:

$$
\mathrm{Z}=\mathrm{A}^{\dagger} \mathrm{W}+\mathcal{N}(\mathrm{A}) \mathrm{C}=\mathrm{A}^{T}\left(\mathrm{AA}^{T}\right)^{-1} \mathrm{~W}+\mathcal{N}(\mathrm{A}) \mathrm{C},
$$

which can be equivalently transformed to:

$$
\mathrm{Z}=\left[\begin{array}{ll}
\mathrm{A}^{T} & \mathcal{N}(\mathrm{A})
\end{array}\right]\left[\begin{array}{c}
\left(\mathrm{AA}^{T}\right)^{-1} \mathrm{~W} \\
\mathrm{C}
\end{array}\right]=\left[\begin{array}{ll}
\mathrm{A}^{T} & \mathcal{N}(\mathrm{A})
\end{array}\right] \mathrm{M}
$$

Note that $\mathrm{A}^{T}$ and $\mathcal{N}(\mathrm{A})$ are orthogonal space to each other. Meanwhile, $A^{T}$ and $\mathcal{N}(\mathrm{A})$ are all column full rank matrices. Therefore $\left[\mathrm{A}^{T} \mathcal{N}(\mathrm{A})\right] \in \mathbb{R}^{n \times n}$ is a full rank matrix and $\operatorname{rank}(\mathrm{Z})=\operatorname{rank}(\mathrm{M})$. As we have analyzed $\operatorname{rank}\left(\left(\mathrm{AA}^{T}\right)^{-1} \mathrm{~W}\right)=$ $\operatorname{rank}(\mathrm{W})=r$, to make $\mathrm{Z}$ lie in rank- $r$ subspace, i.e. $\operatorname{rank}(\mathrm{M})=r$, $\mathrm{C}$ has to lie in the row space of $\left(\mathrm{AA}^{T}\right)^{-1} \mathrm{~W}, \mathrm{C}=\mathrm{D}\left(\mathrm{AA}^{T}\right)^{-1} \mathrm{~W}$, where $\mathrm{D} \in \mathbb{R}^{(n-m) \times m}$ is an arbitrary matrix.

Therefore, we can conclude that for rank-minimization formulation P1, there are always multiple solutions lying in an affine subspace:

$$
\mathrm{Z}=\mathrm{A}^{T}\left(\mathrm{AA}^{T}\right)^{-1} \mathrm{~W}+\mathcal{N}(\mathrm{A}) \mathrm{D}\left(\mathrm{AA}^{T}\right)^{-1} \mathrm{~W} .
$$

b) Frobenius norm minimization.: Denote the SVD of $\mathrm{Z}^{*}$ as $\mathrm{Z}^{*}=\mathrm{U}_{1} \mathrm{D}_{1} \mathrm{~V}_{1}^{T}$, the SVD of $\mathcal{N}(\mathrm{A}) \mathrm{C}$ as $\mathcal{N}(\mathrm{A}) \mathrm{C}=\mathrm{U}_{2} \mathrm{D}_{2} \mathrm{~V}_{2}^{T}$. Note that $\mathrm{A}^{T}$ and $\mathcal{N}(\mathrm{A})$ are orthogonal spaces to each other, i.e. $\mathrm{U}_{1}$ and $\mathrm{V}_{1}$ are orthogonal to $\mathrm{U}_{2}$ and $\mathrm{V}_{2}$ respectively. Thus the SVD of the general solution $\mathrm{Z}=\mathrm{A}^{T}\left(\mathrm{AA}^{T}\right)^{-1} \mathrm{~W}+$ $\mathcal{N}(\mathrm{A}) \mathrm{D}\left(\mathrm{AA}^{T}\right)^{-1} \mathrm{~W}$ can be expressed as:

$$
\mathrm{Z}=\left[\begin{array}{ll}
\mathrm{U}_{1} & \mathrm{U}_{2}
\end{array}\right]\left[\begin{array}{ll}
\mathrm{D}_{1} & \\
& \mathrm{D}_{2}
\end{array}\right]\left[\begin{array}{l}
\mathrm{V}_{1}^{T} \\
\mathrm{~V}_{2}^{T}
\end{array}\right] .
$$

We now show that within the general solutions $\mathrm{Z}=$ $\mathrm{A}^{T}\left(\mathrm{AA}^{T}\right)^{-1} \mathrm{~W}+\mathcal{N}(\mathrm{A}) \mathrm{D}\left(\mathrm{AA}^{T}\right)^{-1} \mathrm{~W}$, the pseudo-inverse solution $\mathrm{Z}^{*}=\mathrm{A}^{T}\left(\mathrm{AA}^{T}\right)^{-1} \mathrm{~W}$ achieves Frobenius norm minimization and nuclear norm minimization.

The Frobenius norm of all the solutions $\|\mathrm{Z}\|_{F}^{2}=\sum \sigma_{i 1}^{2}+$ $\sum \sigma_{i 2}^{2}$, where $\sigma_{i 1}$ and $\sigma_{i 2}$ are diagonal elements of $\mathrm{D}_{1}$ and $\mathrm{D}_{2}$ respectively. Apparently, when $\mathrm{D}=0$, i.e. the pseudoinverse solution $\mathrm{Z}^{*}=\mathrm{A}^{T}\left(\mathrm{AA}^{T}\right)^{-1} \mathrm{~W}$ achieves Frobenius norm minimization.

The nuclear norm of all the solutions $\|\mathrm{Z}\|_{*}=\sum \sigma_{i 1}+$ $\sum \sigma_{i 2}$. By letting $\mathrm{D}=0$, we see that the pseudo-inverse solution i.e. $\mathrm{Z}^{*}=\mathrm{A}^{T}\left(\mathrm{AA}^{T}\right)^{-1} \mathrm{~W}$ achieves nuclear norm minimization.

\section{E. Least-Squares under fixed-rank condition}

In the above section, we give the explicit form of the rank minimization solution subspace. In real world application, we have estimation of the model complexity, i.e., the rank of the matrix to recover, therefore, we obtain a fixed rank least squares problem. The problem to solve is formulated as:

$$
\min _{\mathrm{Z}}\|\mathrm{W}-\mathrm{AZ}\|_{F}^{2}, \text { s.t. } \operatorname{rank}(\mathrm{Z}) \leq k .
$$

Xiang and etc [28] gives closed form solution to above problem as:

1) Perform SVD of $\mathrm{A}$ as $\mathrm{A}=\mathrm{UDV}^{T}$;

2) Extract the first $r$ rows of $\mathrm{U}^{T} \mathrm{~W}$ and denote it as $\mathrm{W}_{r}$;

3) $\quad \mathrm{Z}=\mathrm{V}\left[\begin{array}{c}\mathrm{D}_{r}^{-1} \mathcal{P}_{k}\left(\mathrm{~W}_{r}\right) \\ 0\end{array}\right]$, where $\mathcal{P}_{k}$ denotes a projection onto a set of matrices whose ranks are no more than $k$ (through SVD).

Apparently, the fixed rank least squares solutions are not unique, we can easily extend our analysis for rank minimization subspace and obtain the solution subspace as:

$$
\mathrm{Z}=\mathrm{V}\left[\begin{array}{c}
\mathrm{D}_{r}^{-1} \mathcal{P}_{k}\left(\mathrm{~W}_{r}\right) \\
\mathrm{CD}_{r}^{-1} \mathcal{P}_{k}\left(\mathrm{~W}_{r}\right)
\end{array}\right],
$$

where $\mathrm{C} \in \mathbb{R}^{(n-r) \times r}$ is an arbitrary matrix, $r$ is the rank of $\mathrm{A}$.

Furthermore, if we decompose matrix $\mathrm{V}$ to $\mathrm{V}=\left[\mathrm{V}_{1} \mid \mathrm{V}_{2}\right]$ accordingly, we will have all the rank- $r$ solutions expressed as:

$$
\mathrm{Z}=\mathrm{V}_{1} \mathrm{D}_{r}^{-1} \mathcal{P}_{k}\left(\mathrm{~W}_{r}\right)+\mathrm{V}_{2} \mathrm{CD}_{r}^{-1} \mathcal{P}_{k}\left(\mathrm{~W}_{r}\right),
$$

as $\mathrm{V}_{1}$ and $\mathrm{V}_{2}$ are orthogonal subspace to each other, we can easily prove that when $\mathrm{C}=0$, the resultant $\mathrm{Z}$ achieves nuclear norm minimization and Frobenius norm minimization. 


\section{REGULARIZING THE SOLUTION SPACE}

So far we have characterized the solution subspace of a general rank-minimization (or fixed rank) problem under affine condition $\mathrm{W}=\mathrm{AZ}$.

In this section we will demonstrate how to better regularize (rectify) the solution subspace in order to obtain solutions with better or desired properties. For instance, in solving many computer vision problems, having certain smoothness (or sparseness) property is often desirable. In below, we focus on the rank minimization solution subspace, though our conclusion and analysis are applicable to the fixed-rank solution space as well.

\section{A. Joint low-rank and smoothness optimizations}

First we show how to find smooth solution in the low rank subspace. To measure the smoothness of a solution $\mathrm{Z}$, we use $\mathcal{C}=\|\mathrm{HZ}\|_{F}^{2}$, where $\mathrm{H}$ is a given matrix to characterize the smoothness ( First order, second order and other forms can all be used).

As we have illustrated, the rank minimization solution subspace of $\mathrm{W}=\mathrm{AZ}$ is expressed as:

$$
\mathrm{Z}=\mathrm{A}^{T}\left(\mathrm{AA}^{T}\right)^{-1} \mathrm{~W}+\mathcal{N}(\mathrm{A}) \mathrm{D}\left(\mathrm{AA}^{T}\right)^{-1} \mathrm{~W} .
$$

For each solution in the subspace, the smoothness is derived as:

$$
\begin{aligned}
\|\mathrm{HZ}\|_{F}^{2} & =\left\|\mathrm{HA}^{T}\left(\mathrm{AA}^{T}\right)^{-1} \mathrm{~W}+\mathrm{H} \mathcal{N}(\mathrm{A}) \mathrm{D}\left(\mathrm{AA}^{T}\right)^{-1} \mathrm{~W}\right\|_{F}^{2} \\
& =\operatorname{tr}\left(\mathrm{W}^{T}\left(\mathrm{AA}^{T}\right)^{-T} \mathrm{AH}^{T} \mathrm{HA}^{T}\left(\mathrm{AA}^{T}\right)^{-1} \mathrm{~W}\right) \\
& +\operatorname{tr}\left(\mathrm{W}^{T}\left(\mathrm{AA}^{T}\right)^{-T} \mathrm{D}^{T} \mathcal{N}(\mathrm{A})^{T} \mathrm{H}^{T} \mathrm{H} \mathcal{N}(\mathrm{A})^{T} \mathrm{D}\left(\mathrm{AA}^{T}\right)^{-1} \mathrm{~W}\right) \\
& +2 \operatorname{tr}\left(\mathrm{W}^{T}\left(\mathrm{AA}^{T}\right)^{-T} \mathrm{D} \mathcal{N}(\mathrm{A})^{T} \mathrm{H}^{T} \mathrm{HA}^{T}\left(\mathrm{AA}^{T}\right)^{-1} \mathrm{~W}\right) .
\end{aligned}
$$

As the cost function is a second order function defined in linear subspace, we can even derive a closed-form solution. Let $\frac{\partial\|\mathrm{HZ}\|_{F}^{2}}{\partial \mathrm{D}}=0$, we reach the following normal equation:

$$
\mathcal{N}(\mathrm{A})^{T} \mathrm{H}^{T} \mathrm{H}\left(\mathcal{N}(\mathrm{A}) \mathrm{D}+\mathrm{A}^{T}\right)\left(\mathrm{AA}^{T}\right)^{-1} \mathrm{WW}^{T}\left(\mathrm{AA}^{T}\right)^{-T}=0 .
$$

By introducing Kronecker product and vectorization operator $\operatorname{vec}()$, the above equation is reformulated as:

$\left(\left(\left(\mathrm{AA}^{T}\right)^{-1} \mathrm{WW}^{T}\left(\mathrm{AA}^{T}\right)^{-T}\right)^{T} \otimes\left(\mathcal{N}(\mathrm{A})^{T} \mathrm{H}^{T} \mathrm{H}\right)\right) \operatorname{vec}\left(\mathcal{N}(\mathrm{A}) \mathrm{D}+\mathrm{A}^{T}\right)=0$.

Therefore $\operatorname{vec}\left(\mathcal{N}(\mathrm{A}) \mathrm{D}+\mathrm{A}^{T}\right)$ lies in the null space of $\left(\left(\mathrm{AA}^{T}\right)^{-1} \mathrm{WW}^{T}\left(\mathrm{AA}^{T}\right)^{-T}\right)^{T} \otimes\left(\mathcal{N}(\mathrm{A})^{T} \mathrm{H}^{T} \mathrm{H}\right)$. Denote $\mathcal{N}$ as the null space of $\left(\left(\mathrm{AA}^{T}\right)^{-1} \mathrm{WW}^{T}\left(\mathrm{AA}^{T}\right)^{-T}\right)^{T} \otimes\left(\mathcal{N}(\mathrm{A})^{T} \mathrm{H}^{T} \mathrm{H}\right)$. Then we have:

$$
(\mathrm{I} \otimes \mathcal{N}(\mathrm{A})) \operatorname{vec}(\mathrm{D})+\operatorname{vec}\left(\mathrm{A}^{T}\right)=\mathcal{N} c,
$$

where $c$ expresses the unknown coefficients.

$$
[\mathcal{N}-(\mathrm{I} \otimes \mathcal{N}(\mathrm{A}))]\left[\begin{array}{c}
c \\
\operatorname{vec}(\mathrm{D})
\end{array}\right]=\operatorname{vec}\left(\mathrm{A}^{T}\right)
$$

Thus we can obtain the coefficient matrix $\mathrm{D}$ and solution $\mathrm{Z}=\mathrm{R}\left(\mathrm{RR}^{T}\right)^{-1} \mathrm{~W}+\mathcal{N}(\mathrm{A}) \mathrm{D}\left(\mathrm{AA}^{T}\right)^{-1} \mathrm{~W}$. Obviously, there are multiple solutions to $\mathrm{D}$ but unique solution of $\mathrm{Z}$. In this way, we have obtained the joint low rank and smooth solution $\mathrm{Z}$.

\section{B. Joint low-rank and sparse optimization}

Above we have shown how to find the smoothest $\mathrm{Z}$ in the rank minimization subspace. The same principle can be naturally extended to deal with other optimization criterion such as finding the joint low rank and sparse solution to linear system $\mathrm{W}=\mathrm{AZ}[29]$.

The sparsity of a matrix can be characterized with the element-wise $L_{1}$ norm defined as:

$$
\|\mathrm{Z}\|_{1}=\left\|\mathrm{A}^{T}\left(\mathrm{AA}^{T}\right)^{-1} \mathrm{~W}+\mathcal{N}(\mathrm{A}) \mathrm{D}\left(\mathrm{AA}^{T}\right)^{-1} \mathrm{~W}\right\|_{1} .
$$

By introducing the vectorization operator and Kronecker operation, we reach:

$$
\begin{aligned}
\operatorname{vec}(\mathrm{Z}) & =\operatorname{vec}\left(\mathrm{A}^{T}\left(\mathrm{AA}^{T}\right)^{-1} \mathrm{~W}+\mathcal{N}(\mathrm{A}) \mathrm{D}\left(\mathrm{AA}^{T}\right)^{-1} \mathrm{~W}\right) \\
& =\operatorname{vec}\left(\mathrm{A}^{T}\left(\mathrm{AA}^{T}\right)^{-1} \mathrm{~W}\right)+\left(\left(\left(\mathrm{AA}^{T}\right)^{-1} \mathrm{~W}\right)^{T} \otimes \mathcal{N}(\mathrm{A})\right) \operatorname{vec}(\mathrm{D})
\end{aligned}
$$

Therefore, minimizing the element-wise $L_{1}$ norm of $\mathrm{Z}$ is equivalent to minimizing the $L_{1}$ norm of $\operatorname{vec}(\mathrm{Z})=$ $\operatorname{vec}\left(\mathrm{A}^{T}\left(\mathrm{AA}^{T}\right)^{-1} \mathrm{~W}\right)+\left(\left(\left(\mathrm{AA}^{T}\right)^{-1} \mathrm{~W}\right)^{T} \otimes \mathcal{N}(\mathrm{A})\right) \operatorname{vec}(\mathrm{D})$. This is standard $\min _{\mathbf{x}}\|A \mathbf{x}+\mathbf{b}\|_{1}$ problem, which can be solved via linear programming.

Thus in this way we have found the joint low rank and sparse solution. We even can define column-wise sparsity optimization metric:

$$
\|\mathrm{Z}\|_{2,1}=\left\|\mathrm{A}^{T}\left(\mathrm{AA}^{T}\right)^{-1} \mathrm{~W}+\mathcal{N}(\mathrm{A}) \mathrm{D}\left(\mathrm{AA}^{T}\right)^{-1} \mathrm{~W}\right\|_{2,1}
$$

\section{APPLICATIONS}

In this section, we apply our analysis to practical computer vision problems, showing practical implications of our theory.

\section{A. Subspace segmentation}

For the subspace segmentation problem, Liu et al. [2] proposed the following low rank representation (LRR) formulation,

$$
\min _{\mathrm{Z}, \mathrm{E}} \operatorname{rank}(\mathrm{Z})+\lambda\|\mathrm{E}\|_{l}, \text { s.t. } \mathrm{X}=\mathrm{AZ}+\mathrm{E},
$$

which targets to recover the "lowest-rank representation" of the data with respect to a dictionary A, where $\mathrm{E}$ is the outliers in the data.

Under outlier free scenarios, the low rank representation model falls into the P5 problem and our analysis follows. For the data contaminated by gross error $E$, once we have a rank minimization solution $\mathrm{Z}^{*}$ and corresponding outlier pattern $\mathrm{E}^{*}$, the solution $\mathrm{Z}=\mathrm{Z}^{*}+\mathcal{N}(\mathrm{A}) \mathrm{C}, \mathrm{E}^{*}$ will satisfy the constraint. According to the Theorem 4.3 in [2], the optimal solution to the nuclear norm minimization problem is $Z^{*} \in \operatorname{span}\left(A^{T}\right)$, thus $\mathrm{Z}=\mathrm{Z}^{*}+\mathcal{N}(\mathrm{A}) \mathrm{M}=\mathrm{A}^{T} \mathrm{D}+\mathcal{N}(\mathrm{A}) \mathrm{C}$, when $\mathrm{C}=\mathrm{DM}$, we will obtain a group of solutions achieving rank minimization.

Therefore, we conclude that for the LRR based subspace segmentation problem, even under outliers case, there exists multiple rank minimization solutions. Additionally, as shown in the above section, the nuclear norm minimization solution $\mathrm{Z}^{*}$ achieves Frobenius norm minimization.

SAY SOMETHING ABOUT LATLRR 


\section{B. Matrix completion with missing data}

The affine missing data problems aims to recover a low rank matrix from partial observations, which covers a group of computer vision problems such as video inpainting [4], video denoise [30], repairing low rank texture [3] and etc.

As a special case of the affine missing data problem, recovering low-rank matrix from incomplete measurements is formulated as:

$$
\min \operatorname{rank}(\mathrm{X}) \text {, subject to, } \Omega \odot \mathrm{X}=\mathrm{M} \text {, }
$$

where $\Omega$ is a mask matrix to denote whether the data is observed. This model can easily be transformed to $\operatorname{Avec}(\mathrm{X})=$ $\mathbf{b}$, where $\mathbf{A}$ is a boolean matrix defined by whether $X_{i j}$ is observed or not, thus falls into the affine rank minimization problem Eq. (1).

The rank function is invariant to column-wise and row-wise reshuffle, thus under some circumspect, the rank minimization may not be sufficient to constrain the solution space, see the Fig.1 for an example.

\section{Rigid structure-from-motion}

Rigid structure and motion recovery under perspective camera model can be formulated as: given the image measurements $\mathrm{M}$, recover the projective depths $\Lambda$, such that the rescaled measurements matrix $\mathrm{W}$ is rank 4 , i.e. ,

$$
\begin{aligned}
& \text { Find } \mathrm{W}, \Lambda, \text { subject to, } \\
& \mathrm{W}=\Lambda \odot \mathrm{M}, \\
& \operatorname{rank}(\mathrm{W})=4 .
\end{aligned}
$$

Angst-Zach-Pollefeys [5] proposed to use generalized trace norm, which can easily incorporate priors on the camera motion and scene structure. Dai-Li-He [8] proposed to use the nuclear norm minimization model.

Only using the rank minimization, it is easy to see there is a $F+N$-dimensional solution space since the camera matrices and the points are projective entities and hence only defined up to scale. this can be fixed by column-wise and row-wise balancing. Even if this ambiguity has been ruled out, there is still cases where there exists multiple projectively distinct reconstructions as discussed in [18] and illustrated in Fig. 2.

SAY SOMETHING ABOUT GENERALIZED RECONSTRUCTION THEOREM

\section{Non-rigid structure-from-motion factorization}

Non-rigid structure from motion aims to simultaneously recover deformable shape $S$ and camera motion $R$ such that $\mathrm{W}=$ RS. Paper [6] proposed to formulate the problem of recovering deformable $\mathrm{S}$ from image measurements $\mathrm{W}$ and recovered camera motion $\mathrm{R}$ as rank minimization problem:

$$
\min \operatorname{rank}\left(\mathrm{S}^{\sharp}\right), \quad \mathrm{W}=\mathrm{RS}, \mathrm{S}^{\sharp}=\left[\begin{array}{lll}
\mathrm{P}_{X} & \mathrm{P}_{Y} \mathrm{P}_{Z}
\end{array}\right]\left(\mathrm{I}_{3} \otimes \mathrm{S}\right) .
$$

The rank minimization model originates from the linear combination model of deformable shapes. The above rank minimization model is then relaxed to nuclear norm minimization model and solved efficiently.

For this problem, Hartley-Vidal [31] analyzed the uniqueness of shape recovery under perspective camera model.
They concluded that "even though there are ambiguities in the reconstruction of shape bases and shape coefficients, the reconstructed shape is actually unique". However, in reaching this conclusion, in their proof they made non-degeneracy assumptions about the structure and the motion. In general, without this generality assumption, based on our theory solving the rank-minimization formulation alone, will inevitably admit multiple solutions, unless extra problem-dependent special structure of the solution is enforced, such as the special block matrix structured as demonstrated in [6] and [31] .

\section{SAY SOMETHING ABOUT MY ANALYSIS}

\section{CONCLUSION AND DISCUSSION}

In this paper, we take a novel perspective to carefully look at the uniqueness issue of the solutions to various lowrank/nuclear-norm formulations in computer vision.

We show that for a large group of rank-minimization problems, there almost surely exist multiple solutions. We give explicit characterization of the solution space, and demonstrate how to utilize this characterization to derive/develop new algorithms that better regularize the space, leading to desired solution or even unique solution.

In addition, we analyze the properties of nuclear-norm formulation, revealing that it also holds certain other properties, besides the low-rank property, which we believe may provide a sound explanation to the recent remarkable success of the nuclear-norm.

While this paper may be mistakenly seen as "pouring cold water" on the recent low-rank fever, we however are very positive and confident in this emerging and promising new method. Low-rank method can be very effective in solving hard problems, as long as sufficient care is given, and appropriate domain-specific prior knowledge is applied.

\section{ACKNOWLEDGMENT}

The research is funded in part by Australian Research Council through: DE140100180, DP120103896, DP130104567, LP100100588, and CE140100016.

\section{REFERENCES}

[1] B. Recht, M. Fazel, and P. A. Parrilo, "Guaranteed minimum-rank solutions of linear matrix equations via nuclear norm minimization," SIAM Review, vol. 52, no. 3, pp. 471-501, 2010.

[2] G. Liu, Z. Lin, S. Yan, J. Sun, Y. Yu, and Y. Ma, "Robust recovery of subspace structures by low-rank representation," IEEE Transactions on Pattern Analysis and Machine Intelligence, vol. 35, no. 1, pp. 171-184, Jan 2013.

[3] X. Liang, X. Ren, Z. Zhang, and Y. Ma, "Repairing sparse low-rank texture," in ECCV, 2012, pp. 482-495.

[4] T. Ding, M. Sznaier, and O. Camps, "A rank minimization approach to video inpainting," in $I C C V, 2007$, pp. 1-8.

[5] R. Angst, C. Zach, and M. Pollefeys, "The generalized trace-norm and its application to structure-from-motion problems," in ICCV, 2011, pp. 2502-2509.

[6] Y. Dai, H. Li, and M. He, "A simple prior-free method for non-rigid structure-from-motion factorization," in CVPR, 2012, pp. $2018-2025$.

[7] E. Candès, X. Li, Y. Ma, and J. Wright, "Robust principal component analysis?" J. ACM, vol. 58, no. 3, pp. 11:1-37, 2011.

[8] Y. Dai, H. Li, and M. He, "Element-wise factorization for $\mathrm{N}$-view projective reconstruction," in ECCV, 2010, pp. 396-409. 
[9] — "A simple prior-free method for non-rigid structure-from-motion factorization," International Journal of Computer Vision, vol. 107, no. 2, pp. 101-122, 2014.

[10] Z. Zhang, A. Ganesh, X. Liang, and Y. Ma, "Tilt: Transform invariant low-rank textures," Int. J. Comput. Vision, vol. 99, pp. 1-24, 2012.

[11] Y. Peng, A. Ganesh, J. Wright, W. Xu, and Y. Ma, "RASL: Robust alignment by sparse and low-rank decomposition for linearly correlated images," IEEE Trans. PAMI, vol. 34, no. 11, pp. 2233 -2246, 2012.

[12] Z. Zhang, Y. Matsushita, and Y. Ma, "Camera calibration with lens distortion from low-rank textures," in CVPR, 2011, pp. 2321-2328.

[13] J.-Y. Lee, Y. Matsushita, B. Shi, I. S. Kweon, and K. Ikeuchi, "Radiometric calibration by rank minimization," IEEE Transactions on Pattern Analysis and Machine Intelligence, vol. 35, no. 1, pp. 144-156, 2013.

[14] L. Wu, A. Ganesh, B. Shi, Y. Matsushita, Y. Wang, and Y. Ma, "Robust photometric stereo via low-rank matrix completion and recovery," in ACCV, 2010, pp. 703-717.

[15] M. Ayazoglu, M. Sznaier, and O. Camps, "Euclidean structure recovery from motion in perspective image sequences via hankel rank minimization," in ECCV, 2010, pp. 71-84.

[16] R. Orsi, U. Helmke, and J. B. Moore, "A Newton-like method for solving rank constrained linear matrix inequalities," Automatica, vol. 42, no. 11, pp. 1875-1882, 2006.

[17] K. Jia, T.-H. Chan, Z. Zeng, G. Wang, T. Zhang, and Y. Ma, "ROML: A robust feature correspondence approach for matching objects in a set of images," CoRR, vol. abs/1403.7877, 2014.

[18] R. Hartley and F. Kahl, "Critical configurations for projective reconstruction from multiple views," Int. J. Comput. Vision, vol. 71, no. 1, pp. 5-47, 2007.

[19] B. Nasihatkon, R. Hartley, and J. Trumpf, "A generalized projective reconstruction theorem," preprint, 2013. [Online]. Available: http://users.cecs.anu.edu.au/ u4716751/depth-const.pdf

[20] H. Zhang, Z. Lin, and C. Zhang, "A counterexample for the validity of using nuclear norm as a convex surrogate of rank," in Machine Learning and Knowledge Discovery in Databases, 2013, pp. 226-241.

[21] C.-Y. Lu, H. Min, Z.-Q. Zhao, L. Zhu, D.-S. Huang, and S. Yan, "Robust and efficient subspace segmentation via least squares regression," in ECCV, 2012, vol. 7578, pp. 347-360.

[22] X. Shi and P. S. Yu, "Limitations of matrix completion via trace norm minimization," SIGKDD Explor. Newsl., vol. 12, no. 2, pp. 16-20, 2011.

[23] Y. Ni, J. Sun, X. Yuan, S. Yan, and L.-F. Cheong, "Robust lowrank subspace segmentation with semidefinite guarantees," in IEEE International Conference on Data Mining Workshops, 2010, pp. 11791188 .

[24] Y. Eldar, D. Needell, and Y. Plan, "Uniqueness conditions for lowrank matrix recovery," Applied and Computational Harmonic Analysis, vol. 33, no. 2, pp. $309-314,2012$.

[25] S. Oymak and B. Hassibi, "New Null Space Results and Recovery Thresholds for Matrix Rank Minimization," ArXiv e-prints, 2010.

[26] T. K. Pong, P. Tseng, S. Ji, and J. Ye, "Trace norm regularization: Reformulations, algorithms, and multi-task learning," SIAM J. on $\mathrm{Op}$ timization, vol. 20, no. 6, pp. 3465-3489, Dec. 2010.

[27] A. J. Laub, Matrix Analysis For Scientists And Engineers. Philadelphia, PA, USA: Society for Industrial and Applied Mathematics, 2004.

[28] S. Xiang, Y. Zhu, X. Shen, and J. Ye, "Optimal exact least squares rank minimization," in ACM SIGKDD international conference on Knowledge discovery and data mining, 2012, pp. 480-488.

[29] E. Richard, P.-A. Savalle, and N. Vayatis, "Estimation of simultaneously sparse and low rank matrices," in ICML, 2012, pp. 1351-1358.

[30] H. Ji, C. Liu, Z. Shen, and Y. Xu, "Robust video denoising using low rank matrix completion," in CVPR, 2010, pp. 1791-1798.

[31] R. Hartley and R. Vidal, "Perspective nonrigid shape and motion recovery," in ECCV, 2008, pp. 276-289. 